\title{
Evolution and identity of synapsid carpal bones
}

Susanna Kümmell, Fernando Abdala, Judyth Sassoon, and Virginia Abdala

Acta Palaeontologica Polonica 65 (4), 2020: 649-678 doi:https://doi.org/10.4202/app.00709.2019

To date there is little information on carpal bone homology in late Palaeozoic and Mesozoic Synapsida. Crucial to the understanding of homology in synapsid carpal elements is the fact that different nomenclatures are used for the carpals of non-mammaliamorph Synapsida (Gegenbauer's canonical nomenclature) and Mammaliaformes (mammalian nomenclature). The homologies of the carpals of non-mammaliamorph synapsids and mammals were established early last century and have not been reviewed since then. Here we provide a detailed study of the carpal bones of synapsids ranging in age from the early Permian to Late Cretaceous. The mammaliamorph lunate, previously considered the homologue of the intermedium of non-mammaliamorph synapsids, is interpreted here as homologous to their lateral centrale. We interpret the single mammaliamorph centrale as a homologue of the medial centrale of non-mammaliamorph synapsids. In some synapsid specimens, we found that one or two centralia are fused to the radiale (e.g., the gorgonopsian Arctognathus and tritylodontid Bienotheroides), supporting a digging habit. A third centrale is present in the therocephalian Theriognathus, very likely an abnormal duplication. An additional medial bone in a biarmosuchian was interpreted as a prepollex/ sesamoid. A cartilaginous prepollex/sesamoid may also have been present in several non-mammaliamorph synapsids, which have an open space proximal to distal carpal I. Distal carpal V is completely lost in dicynodonts and it is mainly fused to distal carpal IV in the adult stage of most other therapsid groups, but showed a delayed development in most non-mammaliamorph cynodonts. In mammaliamorphs, distal carpal V is not present. Our observations provide an updated revision of synapsid carpal homologies, mainly on the basis of position and anatomical contacts and also taking into account the results of embryological studies.

Key words: Synapsida, carpus, intermedium, lunate, manus, homology, Permian, Mesozoic.

Susanna Kümmell [susanna.kuemmell@uni-wh.de], Institute of Evolutionary Biology and Morphology, Center for Biomedical Education and Research (ZBAF), Faculty of Health, School of Medicine, University Witten/Herdecke, Stockumer Straße 10, 58454 Witten, Germany. Fernando Abdala [1viutiabdala2@gmail.com ], Unidad Ejecutora Lillo (CONICET-Fundación Miguel Lillo), Tucumán, Argentina and Evolutionary Studies Institute, University of the Witwatersrand, South Africa. Judyth Sassoon [js7892@bristol.ac.uk], School of Earth Sciences, University of Bristol, Queen's Road, Bristol, BS8 1RJ, UK. 
Virginia Abdala [virginia@webmail.unt.edu.ar], Instituto de Biodiversidad Neotropical, CONICET-Universidad Nacional de Tucumán, Catedra de Biologia General, Facultad de Ciencias Naturales e IML, Tucumán, Argentina.

This is an open-access article distributed under the terms of the Creative Commons Attribution License (for details please see creativecommons.org), which permits unrestricted use, distribution, and reproduction in any medium, provided the original author and source are credited.

FoF Full text $(3,055.0 \mathrm{kB})$ ।

Farif Supplementary file $(1,679.3 \mathrm{kB})$ 